\title{
An Exploration of Substrate Coupling at K-Band Between a SiGe HBT Power Amplifier and a SiGe HBT Voltage-Controlled-Oscillator
}

\author{
Jonathan P. Comeau, Member, IEEE, Laleh Najafizadeh, Student Member, IEEE,
} Joel M. Andrews, Student Member, IEEE, A. P. Gnana Prakash, Member, IEEE, and John D. Cressler, Fellow, IEEE

\begin{abstract}
This work presents a case study of circuit-to-circuit substrate coupling between a $24-\mathrm{GHz}$ power amplifier (PA) and a 23-GHz voltage-controlled oscillator (VCO) implemented in a commercially-available SiGe heterojunction bipolar transistor BiCMOS technology. The concurrent operation of these two circuits on the same silicon die results in $-33 \mathrm{~dB}$ of coupling between the PA's output and the VCO's output. Different testing configurations are considered to verify the dominant path of the coupling. These results highlight the potential challenges for silicon-based monolithic systems targeting microwave operational frequencies.
\end{abstract}

Index Terms-Heterojunction bipolar transistor (HBT), power amplifier (PA), voltage-controlled oscillator (VCO).

\section{INTRODUCTION}

QILICON-GERMANIUM (SiGe) heterojunction bipolar transistor (HBT) technology is rapidly entering the world of microwave and millimeter wave systems [1], [2]. The technology offers high speed, low noise SiGe HBTs, while maintaining the high volume, low cost fabrication, and integration capabilities associated with conventional silicon manufacturing [3], [4]. However, as systems expand to higher frequencies of operation and higher levels of integration, the potential for undesired coupling between circuits via the substrate also increases, creating new challenges for system design and evaluation.

Previous studies investigating substrate coupling have examined the characterization and modeling of the substrate [5], [6], highlighting the fact that this problem will only become more challenging at higher frequencies. Other work has also examined individual circuit sensitivities to substrate noise [7]-[9], as well as the potential for circuit-to-circuit coupling between digital/baseband circuits and microwave circuits [10]. However, there has only been limited work examining the potential for coupling to occur between two circuits operating at microwave frequencies in a silicon-based system.

This work focuses on the potential for substrate coupling in $\mathrm{K}$-band systems, and in particular between a $24-\mathrm{GHz}$ power amplifier (PA) and a 23-GHz voltage-controlled oscillator

Manuscript received October 30, 2006; revised February 4, 2007.

The authors are with the School of Electrical and Computer Engineering, Georgia Institute of Technology, Atlanta, GA 30332-0250 USA (e-mail: comeau.jon@gmail.com).

Color versions of one or more of the figures in this letter are available online at http://ieeexplore.ieee.org.

Digital Object Identifier 10.1109/LMWC.2007.895703

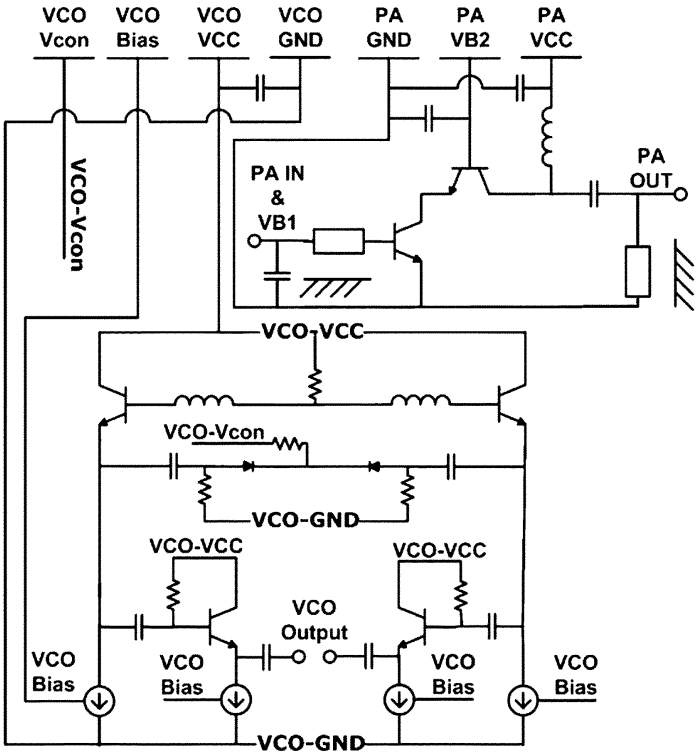

Fig. 1. Schematics of the 24-GHz power amplifier and the 23-GHz VCO fabricated on the same die.

(VCO), when fabricated on the same silicon die and operating concurrently. Various testing configurations are considered to verify that the dominant path of the coupling is through the substrate. The frequency response of the coupling is also examined, demonstrating the impact of the VCO's loop gain on the magnitude of the coupling.

\section{SiGe HBT BICMOS TEChNOLOGY}

The SiGe BiCMOS technology used in this study is the commercially-available Jazz SiGe-120 process, which offers a maximum unity-gain cut-off frequency $\left(f_{T}\right)$ of $150 \mathrm{GHz}$ for the SiGe HBTs [11]. This SiGe platform is fabricated on a conventional $8-10 \Omega$-cm p-type bulk substrate, and contains no additional epi-layer.

\section{Circuit Description}

A 24-GHz SiGe PA design has been incorporated with a 23-GHz SiGe VCO design (Fig. 1), such that both circuits can be biased and measured simultaneously in order to demonstrate the potential for substrate coupling between two microwave circuits. The 24-GHz PA is based on the same topology, design, and layout as presented in [12], which demonstrated a small-signal gain of $12 \mathrm{~dB}$, a return loss greater than $9 \mathrm{~dB}$ on 


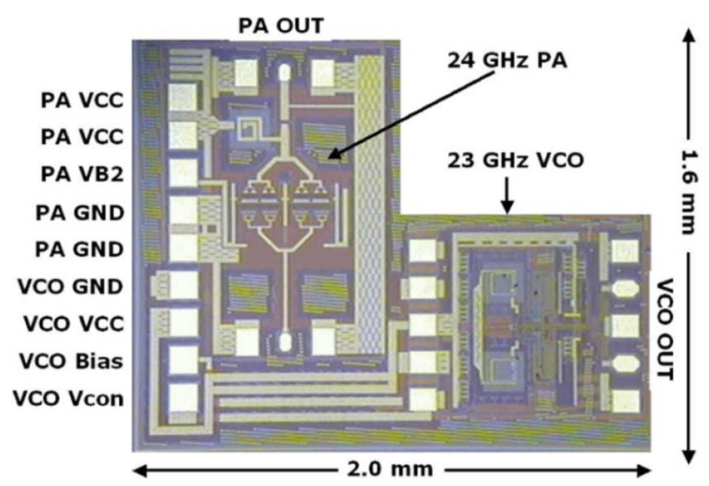

Fig. 2. Die photo of the 24-GHz power amplifier and the 23-GHz VCO fabricated on the same die.

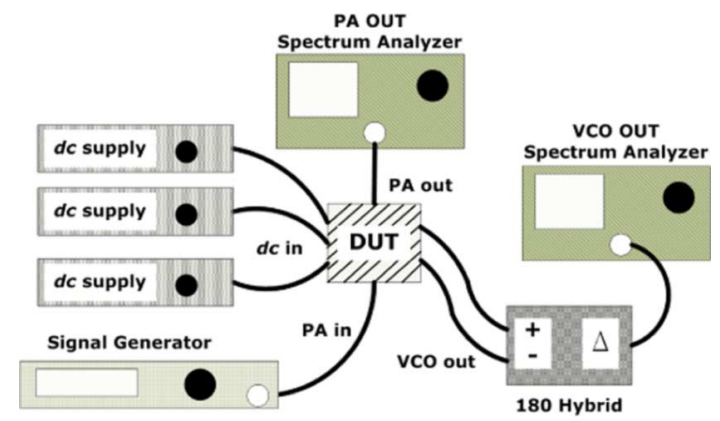

Fig. 3. Block diagram of setup for testing of the VCO and the PA.

both ports, and an $O P_{1 \mathrm{~dB}}$ of $20 \mathrm{dBm}$. The $\mathrm{VCO}$ is based on the same topology, design, and layout as the inductor-based VCO presented in [7], which demonstrated an oscillation frequency of $22.6 \mathrm{GHz}$ and a phase noise of $-104 \mathrm{dBc} / \mathrm{Hz}$ at $1-\mathrm{MHz}$ offset. The circuits were fabricated using the Jazz SiGe-120 [11] technology (Fig. 2), with the center of the power amplifier located approximately $1 \mathrm{~mm}$ from the center of the VCO.

Both the power amplifier and the VCO were given their own $V_{\mathrm{CC}}$, GND, and bias traces and pads, eliminating any form of metalized connection between the two circuits. The circuits were tested at wafer-level on bare die which were approximately 11 mils thick and with no backside metalization. No additional guard-rings or deep trenches were incorporated in the layout of either circuit. However, numerous substrate contacts were incorporated in both designs and connected to the GND buses of the circuits, in an attempt to provide the lowestimpedance radio frequency (RF) connection to the substrate.

\section{EXPERIMENT}

Testing was performed in a shielded room with two spectrum analyzers, a signal generator, and several dc power supplies, as shown in Fig. 3. The output of the VCO was taken differentially and converted to a single-ended signal via a $180^{\circ}$ hybrid. The cable and external hybrid losses were accounted for in the measurement results, and all of the power levels were referenced to the spectrum analyzer connected to the VCO output. The dc bias lines of the circuit were provided via a GGB multicontact wedge [13] with decoupling capacitors between the pins and ground. The die was also tested with a printed circuit board (PCB) evaluation board, with the dc lines wire-bonded out to the board and connected to external decoupling capacitors.

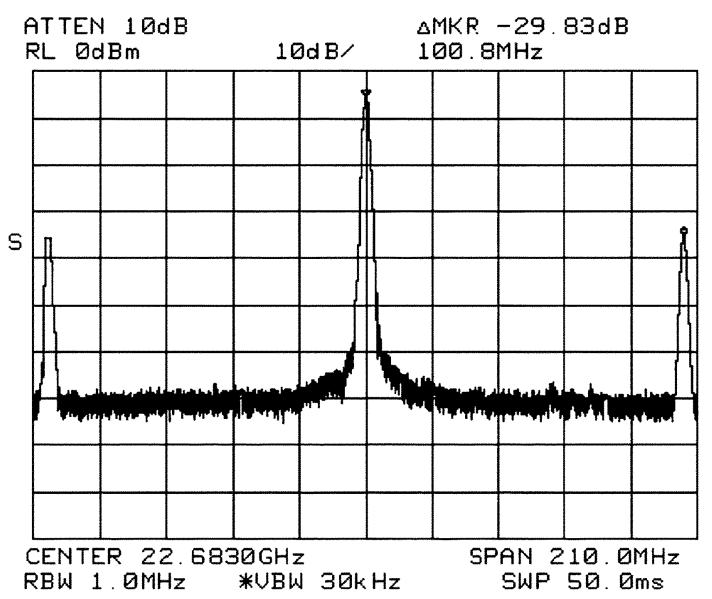

Fig. 4. Spectrum photo of the VCO output when the VCO and the PA are operating at the same time and on the same die.

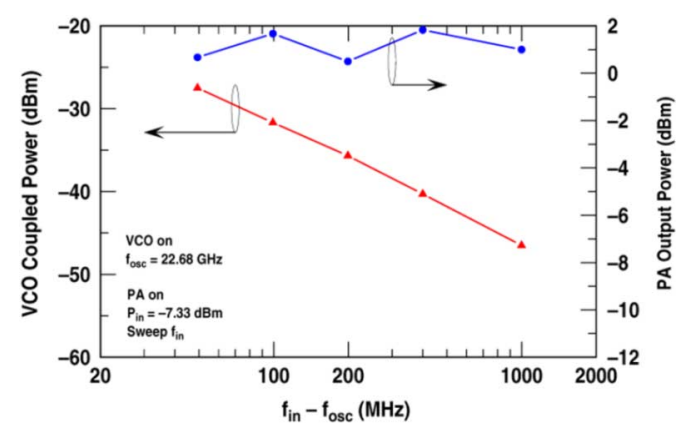

Fig. 5. Coupled power measured at the VCO output and the PA output power versus the frequency difference between $f_{\text {osc }}$ and $f_{\text {in }}$.

The nominal testing configuration for the power amplifier consisted of a 5.0-V bias applied to the $V_{\mathrm{CC}}, 1.8 \mathrm{~V}$ applied to $V_{B 2}$, and $0.865 \mathrm{~V}$ applied to $V_{B 1}$, resulting in a PA $I_{\mathrm{CC}}$ current of $43 \mathrm{~mA}$. An input signal was also applied to the input of the PA, with an input frequency $\left(f_{\text {in }}\right)$ of $22.78 \mathrm{GHz}$ and an input power level $\left(P_{\text {in }}\right)$ of $-7.8 \mathrm{dBm}$, resulting in a PA output power of $2.0 \mathrm{dBm}$. The $\mathrm{VCO}$ was biased from a $3.0-\mathrm{V}$ supply and an input reference current of $1.55 \mathrm{~mA}$ was used to bias the circuit, resulting in a total VCO current of $22 \mathrm{~mA}$. The control voltage of the $\mathrm{VCO}$ was also biased to $3.0 \mathrm{~V}$, resulting in an oscillation frequency $\left(f_{\text {osc }}\right)$ of $22.68 \mathrm{GHz}$ and an output power of $-1.8 \mathrm{dBm}$.

The output spectrum of the VCO can be seen in Fig. 4, demonstrating two $-30 \mathrm{dBc}$ spurs (located at $f_{\text {in }}$ and $\left.2 f_{\text {osc }}-f_{\text {in }}\right)$ in the VCO output. This frequency mixing behavior agrees with the results reported in [7], which demonstrated a VCO's susceptibility to substrate noise by injecting signal into the substrate and monitoring the VCO's output. It should be noted that the VCO used in [7] was based on the same design, layout, and fabrication technology as the VCO used in this study, suggesting that the spurs observed here are also due to noise (or an undesired signal) being sampled from the substrate. Referencing the spur magnitude back to the power amplifier results in $-33 \mathrm{~dB}$ of coupling between the PA output and the VCO output.

The frequency response of this substrate coupling was also characterized (Fig. 5), demonstrating a significant decrease in 
TABLE I

COUPLING RESUlTS FOR DIFFERENT MEASUREMENT CONFIGURATIONS FOR THE PA-TO-VCO COUPLING STUDY

\begin{tabular}{|l|c|c|c|}
\hline Set Up Info & VCO Pout & PA Pout & VCO P Pple \\
\hline & $\mathrm{dBm}$ & $\mathrm{dBm}$ & $\mathrm{dBm}$ \\
\hline VCO ON, PA ON & -1.8 & 2.0 & -31.1 \\
\hline VCO OFF, PA ON & $\mathrm{NA}$ & 1.3 & -59.5 \\
\hline VCO ON, PA OFF & -2.2 & -41.0 & -40.1 \\
\hline $\begin{array}{l}\text { VCO ON, PA OFF } \\
\text { Drive PA Output }\end{array}$ & -2.2 & 2.2 & -47.5 \\
\hline \multicolumn{2}{|r}{ NA $=$ Not Applicable } \\
\hline
\end{tabular}

coupled power as the input frequency of the PA was swept further away from the oscillation frequency of the VCO. As seen in Fig. 5, the PA's output power remains fairly constant over this frequency range, demonstrating that this decrease in coupling is not due to a decrease in PA amplification or output signal.

Different testing setups were also investigated, with the results reported in Table I. In all of these testing configurations, the PA input frequency $\left(f_{\text {in }}\right)$ was set to be $100 \mathrm{MHz}$ higher than the VCO frequency of oscillation $\left(f_{\text {osc }}\right)$. The first row of Table I reports the nominal testing configuration, with the coupled power (VCO $P_{\text {cple }}$ ) representing the magnitude of a single spur present in the VCO output spectrum.

The data in the second row correlates to the setup in which the VCO is unbiased, but with the PA biased and a PA input signal applied. As seen from these results, there is an $18-\mathrm{dB}$ decrease in coupled signal appearing at the VCO output. These results, along with the frequency response performance of the coupling shown in Fig. 5, demonstrate that the undesired signal (which is coupled to the VCO's output) is being amplified by the VCO's internal feedback loop. If the VCO's feedback loop is eliminated (i.e., if the VCO is turned off) or the frequency of the injected signal is out of the VCO's loop-gain bandwidth, the magnitude of the coupled signal is significantly reduced.

A 9- $\mathrm{dB}$ reduction of coupling is also observed when the PA is unbiased, but with the input signal of the PA still applied to the input pad (as reported in the third row of Table I). This result verifies that the coupled signal apparent at the VCO output is not merely due to coupling between the PA input probe and the VCO. The biasing and amplification of the PA significantly increases the coupling between these two circuits.

The final testing configuration examined the possibility of the PA output probe coupling to the VCO. Again the PA was left unbiased, but with PA drive signal now applied directly to the PA output pad (as opposed to the PA input pad). The PA drive signal was also increased by $10 \mathrm{~dB}$ to more accurately model the magnitude of the power present at the PA output for the nominal configuration. As seen in the last row of Table I, the coupled signal power apparent at the VCO output decreases significantly, confirming that the coupling is not simply probe-toprobe or probe-to-VCO.

\section{CONCLUSION}

We have successfully demonstrated the potential for one microwave silicon-based circuit to couple to another via the substrate, when both circuits are operating concurrently and on the same die. The frequency response of this coupling and the form in which it is manifested agrees with previous substrate noise studies. This coupling occurs without any form of metalized connection between the two circuits, eliminating the possibility of cross-talk between supply lines, or signal leakage via shared dc biasing circuitry. Different testing configurations were also examined to scrutinize these effects, verifying that the coupling was not simply probe-to-probe or probe-to-VCO.

These findings highlight the challenges associated with monolithic silicon-based systems operating at frequencies greater than $20 \mathrm{GHz}$. Although some common design practices (such as guard-rings, the use of higher resistivity substrates, and the use of shielded inductors) provide some relief at lower frequencies of operation (i.e., $<10 \mathrm{GHz}$ ), these techniques are not nearly as effective at higher frequencies of operation. Further studies are still needed to fully understand and mitigate these substrate coupling concerns.

\section{ACKNOWLEDGMENT}

The authors are grateful for the assistance and support of R. Van Art, A. Karroy, J. Cordovez, and the SiGe Team at Jazz Semiconductor, as well as, the members of the Georgia Tech Research Institute Fellows Council, Dr. R. Bohlander, and Dr. D. Parekh for the GTRI IRAD project support.

\section{REFERENCES}

[1] A. J. Joseph, D. L. Harame, B. Jagannathan, D. Coolbaugh, D. Ahlgren, J. Magerlein, L. Lanzerotti, N. Feilchenfeld, S. St. Onge, J. Dunn, and E. Nowak, "Status and direction of communication technologies-SiGe BiCMOS and RFCMOS," Proc. IEEE, vol. 93, no. 9, pp. 1539-1558, Sep. 2005.

[2] M. Racanelli and P. Kempf, "SiGe BiCMOS technology for RF circuit applications," IEEE Trans. Electron. Dev., vol. 52, no. 7, pp. 1259-1270, Jul. 2005.

[3] J. D. Cressler and G. Niu, Silicon-Germanium Heterojunction Bipolar Transistors. Boston, MA: Artech House, 2003.

[4] J. D. Cressler, Ed., Silicon Heterostructure Handbook. Boca Raton, FL: CRC, 2006.

[5] W. Steiner, M. Pfost, H.-M. Rein, A. Sturmer, and A. Schuppen, "Methods for measurement and simulation of weak substrate coupling in high-speed bipolar ICs," IEEE Trans. Microw. Theory Tech., vol. 50, no. 7, pp. 1705-1713, Jul. 2002.

[6] K. A. Jenkins, "Substrate coupling noise issues in silicon technology," in Proc. IEEE Top. Meeting Silicon Monolith. Integr. Circuirts RF Syst., 2004, pp. 91-94.

[7] J. P. Comeau and J. D. Cressler, "Microwave VCO susceptibility to substrate noise in a fully-integrated $150 \mathrm{GHz}$ SiGe HBT BiCMOS technology," in IEEE MTT-S Int. Dig., Jun. 2005, pp. 2235-2238.

[8] N. Checka, D. D. Wentzloff, A. Chandrakasan, and R. Reif, "The effect of substrate noise on VCO performance," in Proc. IEEE Rad. Freq. Integ. Circuits Symp., Jun. 2005, pp. 523-526.

[9] C. Soens, G. Van der Plas, P. Wambacq, and S. Donnay, "Performance degradation of an LC-tank VCO by impact of digital switching noise," in Proc. 30th Eur. Solid-State Circuits Conf., Sep. 2004, pp. 119-122.

[10] E. Sonmez, S. Chartier, A. Trasser, and H. Schumacher, "Isolation issues in multifunctional $\mathrm{Si} / \mathrm{SiGe}$ ICs at $24 \mathrm{GHz}$," in IEEE MTT-S Int. Dig., Jun. 2005, pp. 12-17.

[11] M. Racanelli and P. Kempf, "SiGe BiCMOS technology for communication products," in Proc. IEEE Custom Integr. Circuits Conf., 2003, pp. 331-334.

[12] J. P. Comeau, J. M. Andrews, and J. D. Cressler, "A monolithic 24 GHz, $20 \mathrm{dBm}, 14 \%$ PAE SiGe HBT power amplifier," in Proc. 36th Eur. Microw. Conf., Sep. 2006, pp. 419-422.

[13] GGB Industries, Inc., "GGB multi-contact wedge," Tech. Rep., Sep. 2006 [Online]. Available: http://www.ggb.com 